\title{
Occurrence of Burkholderia glumae on Rice and Field Crops in Korea
}

\author{
Jinwoo Kim ${ }^{1}$, Yongsung Kang², Jung-Gun Kim², Okhee Choi ${ }^{2}$ and Ingyu Hwang ${ }^{2 *}$ \\ ${ }^{1}$ Institute of Agriculture and Life Science and Department of Applied Biology, Gyeongsang National University, Jinju 660-701, \\ Korea \\ ${ }^{2}$ Department of Agricultural Biotechnology, Seoul National University, Seoul 151-921, Korea \\ (Received on July 21, 2010; Accepted on August 16, 2010)
}

\begin{abstract}
Burkholderia glumae causes bacterial rice grain rot and bacterial wilt on many field crops. We developed a simple diagnostic streak method for the isolation of B. glumae from diseased plant material. The geographical distribution of 178 Korean isolates shows that B. glumae is widely spread in South Korea.
\end{abstract}

Keywords : Burkholderia glumae, bacterial rice grain rot, bacterial wilt

Bacterial grain rot in rice caused by Burkholderia glumae has become a serious problem in Korea, Japan, and other Asian countries. This disease is highly dependent upon weather conditions at the flowering stage (Tsushima et al., 1996). Infected seeds are a primary source of inocula, and secondary infections occur at the flowering stage under high temperatures and moisture levels. Environmental conditions during the summer in Korea are similar to subtropical conditions, which are favorable for development of the disease. The bacterium also causes bacterial wilt on many field crops through the production of phytotoxins that are responsible for major symptom development (Jeong et al., 2003). The bacterium produces a bright yellow pigment that has been identified as a toxoflavin, and which is essential for pathogenicity (Sato et al., 1989; Jeong et al., 2003). The production of toxoflavin, which is dependent on the growth temperature and has antibacterial and antifungal activities, is maximal at $37^{\circ} \mathrm{C}$ but undetectable at $25-28^{\circ} \mathrm{C}$ (Matsuda and Sato, 1988).

For the isolation of B. glumae from diseased plant materials, several diagnostic methods, including serological tests and semi-selective media, have been developed (Choi et al., 1989; Kim et al., 1993). Since the toxoflavin produced by $B$. glumae is toxic to other microbes, including bacteria and fungi, it is not necessary to use surface sterilization or selective media for the isolation. To isolate B. glumae from both xylem tissues of various field crops

\footnotetext{
*Corresponding author.

Phone) +82-2-880-4676, FAX) +82-2-873-2317

E-mail)ingyu@snu.ac.kr
}

and rice grains (Fig. 1A and B), diseased stem tissue was cut into small pieces, surface-sterilized, and placed in an Eppendorf tube containing $1 \mathrm{ml}$ of sterile distilled water (Jeong et al., 2003). The resulting suspension was streaked onto Luria-Bertani (LB) agar plates (10 $\mathrm{g}$ of tryptone, $5 \mathrm{~g}$ of yeast extract, and $5 \mathrm{~g} / \mathrm{l} \mathrm{NaCl}$ ) with a sterile toothpick and incubated at $37^{\circ} \mathrm{C}$. To isolate $B$. glumae from rice grains, the seed coat was first removed from infected seeds using sterile forceps. The un-coated seeds were then chopped with a sterile scalpel in a plastic Petri dish. A small amount of healthy tissue was removed from the most rotted area using a sterile scalpel. The rotted portion was placed on an

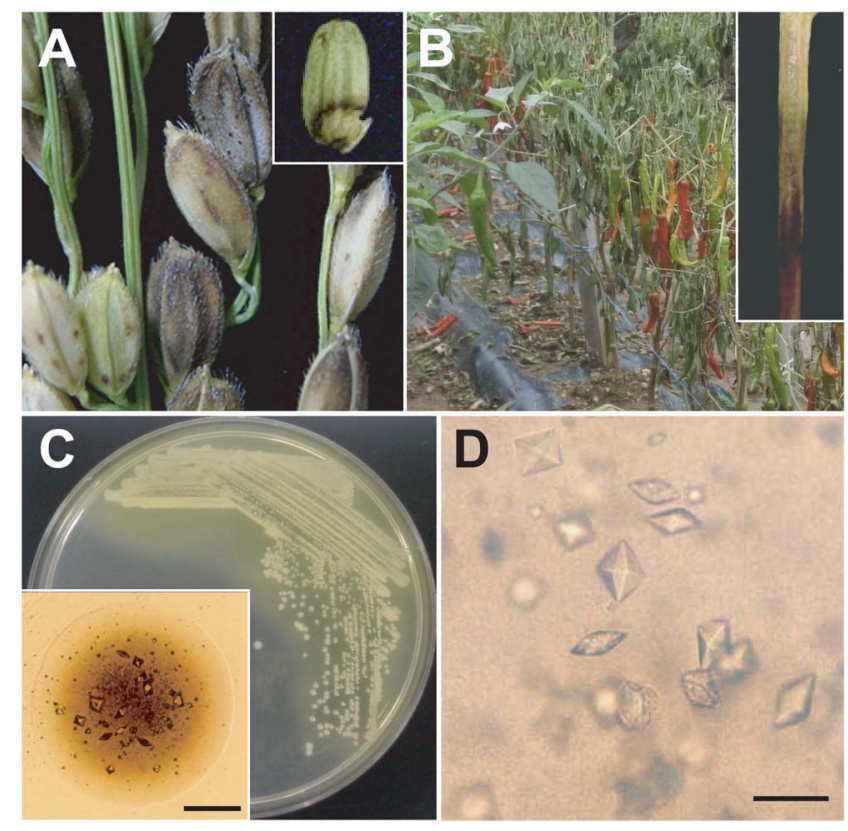

Fig. 1. Symptoms and colony morphological features. A: Typical symptoms of bacterial grain rot in rice caused by Burkholderia glumae. A hulled rice grain in the small box shows discoloration due to toxoflavin. B: Bacterial wilt symptoms caused by $B$. glumae on hot pepper plants. A small box shows disease symptom in xylem tissues of hot pepper 7 days after inoculation of B. glumae. C: A typical yellow pigment was observed due to toxoflavin production by B. glumae on LB agar. A single colony in the small box was filled with small crystals of toxoflavin (bar $=0.5 \mathrm{~mm}$ ). D: Enlarged toxoflavin crystals were observed under microscope $(\times 40)(\mathrm{bar}=0.2 \mathrm{~mm})$. 


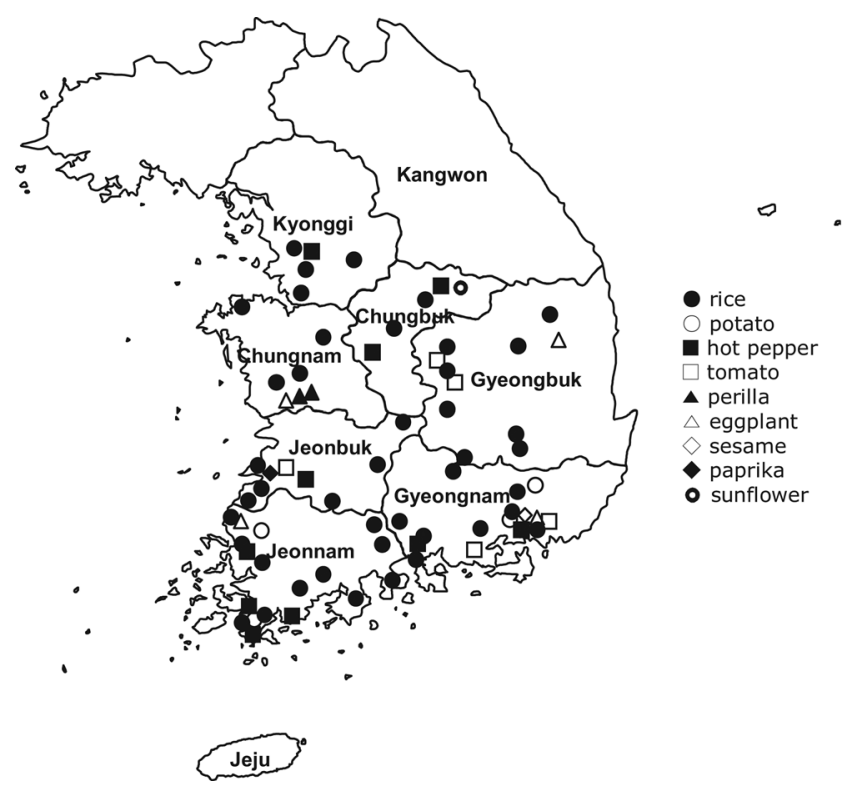

Fig. 2. Locations in Korea where Burkholderia glumae isolates were collected. Symbols indicate representative locations for the same geographical origin and the corresponding host plants that bacteria were isolated from.

LB agar plate and streaked with a sterile toothpick. Streaks were made at right angles in three directions using a new toothpick for each streak. A single colony of B. glumae was found after two days of incubation at $37^{\circ} \mathrm{C}$ (Fig. 1C). This colony was re-streaked on new medium to ensure purity of the culture. A colony of B. glumae was packed with numerous crystals of toxoflavin, which were examined under a microscope (Fig. 1D). Taken together, we describe a simple method for the isolation of B. glumae from diseased plant materials based on crystals, which can be used for the rapid detection of B. glumae.

Fig. 2 shows the geographical distribution of various Korean isolates of B. glumae. A total of 106 bacterial isolates were obtained from diseased crops in seven Korean provinces: Kyonggi, Chungbuk, Chungnam, Jeonbuk, Jeonnam, Gyeongbuk, and Gyeongnam (Jeong et al., 2003). Rice grains with symptoms of grain rot were collected throughout South Korea during the growing season in 2000 and 2006, and 72 isolates were obtained from rice panicles. The panicles exhibited a range of symptoms, varying from slight discoloration to a brown and blotchy appearance. When the isolates were inoculated into rice, panicle dis- coloration appeared after three days (data not shown). The number of diseased panicles increased gradually, and most of the panicles of the rice plants tested were discolored after ten days. All 72 isolates induced hypersensitivity on tobacco plants (data not shown), and nine representative isolates produced toxoflavin based on thin layer chromatography (Jeong et al., 2003).

In a previous report, we suggested that rice panicles infected with B. glumae could serve as a source inoculum for bacterial wilt on field crops, although we have yet to obtain evidence for this. Since cross-infection between field crops and rice occurs when they are cultivated in close proximity during a single growth period, it is necessary to conduct a geographical study of $B$. glumae occurrence to effectively control it.

\section{Acknowledgments}

This work was supported by the Crop Functional Genomics Center of the 21st Century Frontier Research Programs, which is funded by the Ministry of Education, Science and Technology (MEST) of the Republic of Korea.

\section{References}

Choi, J. E., Lee, J. C. and Oh, S. H. 1989. Serological diagnosis of bacterial grain rot of rice. Korean J. Plant Pathol. 5:146-150.

Jeong, Y., Kim, J., Kim, S., Kang, Y., Nagamatsu, T. and Hwang, I. 2003. Toxoflavin produced by Burkholderia glumae causing rice grain rot is responsible for inducing bacterial wilt in many field crops. Plant Dis. 87:890-895.

Kim, H. M., Song, W. Y. and Schaad, W. N. 1993. A semiselective medium for the isolation of Pseudomonas glumae from rice seeds. Korean J. Plant Pathol. 9:248-251.

Matsuda, I. and Sato, Z. 1988. Regulation between pathogenicity and pigment productivity in the causal agent of bacterial grain rot of rice. Ann. Phytopathol. Soc. Jpn. 54:378.

Sato, Z., Koiso, Y., Iwasaki, S. and Matsuda, I. 1989. Toxins produced by Pseudomonas glumae. Ann. Phytopathol. Soc. Jpn. 55:356-359.

Tsushima, S., Naito, H. and Koitabashi, M. 1996. Population dynamics of Pseudomonas glumae, the causal agent of bacterial grain of rice, on leaf sheaths of rice plants in relation to disease development in the field. Ann. Phytopathol. Soc. Jpn. 62:108-113. 